\title{
MULTIPLIERS ON SPACES OF ANALYTIC FUNCTIONS
}

\author{
OSCAR BLASCO
}

\begin{abstract}
In the paper we find, for certain values of the parameters, the spaces of multipliers $(H(p, q, \alpha), H(s, t, \beta))$ and $\left(H(p, q, \alpha), l^{s}\right)$, where $H(p, q, \alpha)$ denotes the space of analytic functions on the unit disc such that $(1-r)^{\alpha} M_{p}(f, r) \in L^{q}\left(\frac{d r}{1-r}\right)$. As corollaries we recover some new results about multipliers on Bergman spaces and Hardy spaces.
\end{abstract}

\section{§0. INTRODUCTION.}

Given two sequence spaces $X$ and $Y$, we denote by $(X, Y)$ the space of multipliers from $X$ into $Y$, that is the space of sequences of complex numbers $\left(\lambda_{n}\right)$ such that $\left(\lambda_{n} a_{n}\right) \in Y$ for $\left(a_{n}\right) \in X$.

When dealing with spaces of analytic functions defined on the open unit disc $D$ we associate to each analytic function $f(z)=\sum_{n=0}^{\infty} a_{n} z^{n}$ the corresponding sequence of Taylor coefficients $\left(a_{n}\right)$. In this sense any space of analytic functions is regarded as a sequence space and it makes sense to study multipliers acting on different classes of spaces such as Hardy spaces, Bergman spaces and so on.

During the last decade lots of results were obtained (see [AS, BST, DS1, M, MP1, MP2, S2, SW]). Recently the interest on similar questions has been renewed and some new results on multipliers on Hardy and Bergman spaces have been achieved (see [W, MP3, JP, MZ, V]).

The aim of this paper is to study spaces of multipliers acting on certain general classes of analytic functions, denoted by $H(p, q, \alpha)$, which consists of functions on the unit disc such that $\left(\int_{0}^{1}(1-r)^{\alpha q-1} M_{p}^{q}(f, r) d r\right)^{1 / q}<\infty$.

The definition of these classes goes back to the work of Hardy and Littlewood (see [HL1,HL2]) and they were intensively studied for different reasons and by several authors. The reader is referred to the papers [DRS, F1, F2, MP1, S1, Sh] for information and properties on the spaces.

There are two different techniques used in the paper. On one hand the use of a general theorem on operators acting on $H(p, q, \alpha)$ for $0<p \leq 1$ which allows us to find $(H(p, q, \alpha), H(s, t, \beta))$ and $\left(H(p, q, \alpha), l^{s}\right)$ for the cases $0<p, q \leq 1$ and $1 \leq s, t \leq \infty$ and also for $0<p \leq 1 \leq q$ although only for particular cases of $s$ and $t$. In particular we can get a proof of the recent theorem, due to M. Mateljevic and

1991 Mathematics Subject Classification. $42 \mathrm{~A} 45$.

Key words and phrases. Multipliers, spaces of analytic functions.

Partially supported by the Spanish DGICYT, Proyectos PB89-0106 and PB92-0699

Typeset by $\mathcal{A}_{\mathcal{M}} \mathcal{S}-\mathrm{T}_{\mathrm{E}} \mathrm{X}$ 
M. Pavlovic (see [MP3]), which says that $\left(H^{1}, B M O\right)=$ Bloch and to realize that this result still has an extension to the setting of $H(p, q, \alpha)$ spaces.

On the other hand, the use of Kintchine's inequality allows us to see that multipliers on $H(p, q, \alpha)$ for values $p \geq 2$ depend in most cases on those for $H(2, q, \alpha)$, being these spaces isomorphic to $l(2, q)$ (see definition below) and hence very easy to deal with them. This will allow us, in particular, to extend an give simpler proofs of the results on Bergman spaces $B^{p}$ (corresponding to $H(p, p, 1 / p)$ ) due to P. Wojtaszczyk (see $[\mathrm{W}]$ ).

The paper is divided into six sections. The first one has a preliminary character and several general facts and properties on the spaces are shown. Sections 2 and 3 are devoted to the cases $H(p, q, \alpha)$ for values $p=2$ and $0<p \leq 1$ respectively, finding useful Littlewood-Paley type characterizations of the spaces and a general theorem on operators acting on $H(p, q, \alpha)$ when $0<p, q \leq 1$. In Section 4 we deal with multipliers $\left(H(p, q, \alpha), l^{s}\right)$ recalling the known results and proving a new one regarding the case $0<p \leq 1 \leq q$. Section 5 concerns with multipliers $(H(p, q, \alpha), H(s, t, \beta))$ and finally we obtain applications to Bergman and Hardy spaces in the last section. Besides the results in $[\mathrm{W}, \mathrm{MP}]$ just pointed out above we can recover some new ones on multipliers acting on Bergman spaces $B^{p}$ for $1 \leq p \leq 2$ obtained in $[\mathrm{MZ}, \mathrm{V}]$

Throughout the paper all functions $f$ will be analytic on the unit disc and $\lambda$ may be considered as a function $\lambda(z)=\sum_{n=0}^{\infty} \lambda_{n} z^{n}$ or as the sequence $\left(\lambda_{n}\right)$ given by its Taylor coefficients. If $f(z)=\sum_{n=0}^{\infty} a_{n} z^{n}$ and $g(z)=\sum_{n=0}^{\infty} b_{n} z^{n}$ then we shall write $f * g(z)=\sum_{n=0}^{\infty} a_{n} b_{n} z^{n}$. As usual $M_{p}(f, r)$ stands for $\left(\int_{0}^{2 \pi}\left|f\left(r e^{i \theta}\right)\right|^{p} \frac{d \theta}{2 \pi}\right)^{1 / p}$ and we denote by $H^{p}$ and $B^{p}$ the classical Hardy and Bergman spaces respectively. Finally recall that the notation $p^{\prime}$ will be used for the conjugate exponent of $p$ verifying $1 / p+1 / p^{\prime}=1$ and $C$ will stand for a constant that may vary from line to line.

\section{§1. BACKGROUND AND PRELIMINARY RESULTS.}

Definition 1.1. Let $0<p \leq \infty$ and $0<\alpha, q<\infty$. $H(p, q, \alpha)$ will be the space of analytic functions on the open unit disc $D$ satisfying

$$
\|f\|_{p, q, \alpha}=\left(\int_{0}^{1}(1-r)^{\alpha q-1} M_{p}^{q}(f, r) d r\right)^{1 / q}<\infty .
$$

Let $0<p \leq \infty$ and $0<\alpha . H_{\alpha}^{p}($ or $H(p, \infty, \alpha))$ will be the space of analytic functions on the open unit disc $D$ satisfying

$$
\|f\|_{p, \infty, \alpha}=\sup _{0<r<1}(1-r)^{\alpha} M_{p}(f, r) d r<\infty .
$$

Let us collect some estimates to be used in the sequel.

Lemma A (General estimates). Let $p_{1} \leq p_{2}, \gamma>1$ and $\delta<\beta$. Then

$$
M_{p_{2}}(f, r) \leq C(1-r)^{1 / p_{2}-1 / p_{1}} M_{p_{1}}(f, r)(\text { see }[\mathrm{D}, \text { page } 84]),
$$




$$
\begin{gathered}
\int_{0}^{2 \pi} \frac{d \theta}{\left|1-z e^{i \theta}\right|^{\gamma}}=O\left(1 /(1-|z|)^{\gamma-1}\right)(\text { see }[\mathrm{D}, \text { page } 65]) \\
\int_{0}^{1} \frac{(1-r)^{\delta-1}}{(1-r s)^{\beta}} d r=O\left(1 /(1-s)^{\beta-\delta}\right)(\text { see }[\mathrm{SW}, \text { Lemma } 6]) .
\end{gathered}
$$

We are going to formulate the results and properties that will be needed later on. We start with some very elementary embeddings which follow easily from (1.1).

Lemma B (Embeddings). If $0<p, q, \alpha, p_{1} \leq p_{2}, q_{1} \leq q_{2}$ and $\alpha_{1} \leq \alpha_{2}$. Then

$$
\begin{gathered}
H^{p_{2}} \subset H\left(p_{2}, q_{1}, \alpha_{1}\right) \subset H\left(p_{1}, q_{2}, \alpha_{2}\right) \subset H_{\alpha_{2}}^{p_{1}}, \\
H\left(p_{1}, q, \alpha\right) \subset H\left(p_{2}, q, \alpha+1 / p_{1}-1 / p_{2}\right), \\
H\left(p, q_{2}, \alpha\right) \subset H\left(p, q_{1}, \alpha+1 / q_{1}-1 / q_{2}\right) .
\end{gathered}
$$

Now we shall state two easy results about multipliers with hold in general cases.

Lemma C (Multipliers). Let $0<p_{1}, p_{2}, q_{1}, q_{2}, \alpha, \beta<\infty, \frac{1}{p_{1}}+\frac{1}{p_{2}} \geq 1, \frac{1}{p}=$ $\frac{1}{p_{1}}+\frac{1}{p_{2}}-1$ and $\frac{1}{q}=\frac{1}{q_{1}}+\frac{1}{q_{2}}$. Then

$$
\|f * g\|_{p, q, \alpha+\beta} \leq\|f\|_{p_{1}, q_{1}, \alpha}\|g\|_{p_{2}, q_{2}, \beta} .
$$

Let $0<q, \alpha<\infty$ and $f(z)=\sum_{n=0}^{\infty} a_{n} z^{n}$. Then

$$
\left(\sum_{n=0}^{\infty} \frac{\left|a_{n}\right|^{q}}{(n+1)^{q \alpha+1}}\right)^{1 / q} \leq C\|f\|_{1, q, \alpha}
$$

Proof. (1.7) follows by applying consecutively Young's and Hölder's inequalities.

To show (1.8) we simply use the trival estimate $\left|a_{n}\right| r^{n} \leq M_{1}(f, r)$.

$$
\begin{aligned}
\|f\|_{1, q, \alpha} & =\left(\sum_{n=1}^{\infty} \int_{1-1 / n}^{1-1 /(n+1)}(1-r)^{q \alpha-1} M_{1}^{q}(f, r) d r\right)^{1 / q} \\
& \geq\left(\sum_{n=1}^{\infty} \int_{1-1 / n}^{1-1 /(n+1)}(1-r)^{q \alpha-1} r^{q n}\left|a_{n}\right|^{q} d r\right)^{1 / q} \\
& \geq\left(\sum_{n=1}^{\infty} \frac{\left|a_{n}\right|^{q}}{(n+1)^{q \alpha-1}} \frac{1}{n(n+1)}\left(1-\frac{1}{n}\right)^{n q}\right)^{1 / q} \\
& \geq C\left(\sum_{0}^{\infty} \frac{\left|a_{n}\right|^{q}}{(n+1)^{q \alpha+1}}\right)^{1 / q} .
\end{aligned}
$$

The interest on these spaces appeared from their conection with Hardy spaces and mainly from inequalities like the ones we mention in the next lemma. They were shown in [HL1, F1, LP]. 
Lemma D (Inequalities). Let $1 \leq p \leq 2 \leq q<\infty$. Then

$$
\begin{aligned}
& \left(\int_{0}^{1}(1-r)^{\frac{p}{2}} M_{2}^{p}\left(f^{\prime}, r\right) d r\right)^{\frac{1}{p}} \leq C\|f\|_{H_{p}}, \\
& \left(\int_{0}^{1}(1-r) M_{p}^{2}\left(f^{\prime}, r\right) d r\right)^{\frac{1}{2}} \leq C\|f\|_{H_{p}}, \\
& \left(\int_{0}^{1}(1-r)^{q-1} M_{q}^{q}\left(f^{\prime}, r\right) d r\right)^{\frac{1}{q}} \leq C\|f\|_{H_{q}} .
\end{aligned}
$$

Remark 1.1. It is easy to see that (1.9) is equivalent to

$$
\left(\int_{0}^{1}(1-r)^{\frac{-p}{2}} M_{2}^{p}(f, r) d r\right)^{\frac{1}{p}} \leq C\|f\|_{H_{p}} .
$$

The two first inequalities are due to Hardy and Littlewood. The case $p=2$ follows from the Plancherel's theorem, the case $p=1$ from factorization of $H^{1}$-functions as product of two $H^{2}$-functions and finally the cases $1<p<2$ from interpolation (see $[\mathrm{D}$, Theorems 5.11, 5.6]). An alternative much simpler proof of (1.9), based upon Marcinkiewich's interpolation, was shown by Flett (see [F1]).

The inequality (1.11) is due to Littlewood an Paley (see [LP]) and it can be obtained by means of the operator $T(f)=(1-|z|) f^{\prime}(z)$. This operator is bounded from $H^{2}$ into $L^{2}\left(\frac{d r}{1-r}, L^{2}(d \theta)\right)$ and from $H^{\infty}$ into $L^{\infty}\left(\frac{d r}{1-r}, L^{\infty}(d \theta)\right)$, then we get the other cases using interpolation.

Given $f(z)=\sum_{n=0}^{\infty} a_{n} z^{n}$ and $\beta>0$ we denote by $f^{(\beta)}$ the fractional derivative of $f$ defined by $f^{(\beta)}(z)=\sum_{n=0}^{\infty} \frac{\Gamma(\beta+n+1)}{\Gamma(\beta) n !} a_{n} z^{n}$.

The reader is referred to [HL1, F1, F2, DRS] for different results on fractional derivatives. Let us recall that for $1 \leq p \leq \infty, 0<\alpha, \beta<\infty$ we have (see [D, Theorem 5.5] for $\beta=1$ )

$$
f \in H_{\alpha}^{p} \text { if and only if } f^{(\beta)} \in H_{\alpha+\beta}^{p} .
$$

Next result cover other values of $0<q<\infty$ and although part of the folklore we include here a proof because of the lack of any reference.

Theorem A (Fractional derivatives). Let $1 \leq p \leq \infty, 0<q, \alpha, \beta<\infty$. Then $f$ belongs to $H(p, q, \alpha)$ if and only if $f^{(\beta)}$ belongs to $H(p, q, \alpha+\beta)$.

Proof. Let us assume $f \in H(p, q, \alpha)$. Denoting by $K_{\beta}(z)=1 /(1-z)^{\beta+1}$ we can write $f^{(\beta)}=f * K_{\beta}$, therefore

$$
M_{p}\left(f^{(\beta)}, r^{2}\right) \leq M_{p}(f, r) M_{1}\left(K_{\beta}, r\right) \leq C \frac{1}{(1-r)^{\beta}} M_{p}(f, r) .
$$


From this easily follows that $f^{(\beta)} \in H(p, q, \alpha+\beta)$.

Conversely, let us assume $f^{(\beta)} \in H(p, q, \alpha+\beta)$ and $\beta \geq 1$. Observe first that

$$
f(z)=\int_{0}^{1}(1-s)^{\beta-1} f^{(\beta)}(s z) d s .
$$

This gives

$$
\begin{aligned}
M_{p}(f, r) & \leq \int_{0}^{1}(1-s)^{\beta-1} M_{p}\left(f^{(\beta)}, r s\right) d s \\
& =r^{-\beta} \int_{0}^{r}(r-s)^{\beta-1} M_{p}\left(f^{(\beta)}, s\right) d s \\
& \leq \int_{0}^{r}(1-s)^{\beta-1} M_{p}\left(f^{(\beta)}, s\right) d s
\end{aligned}
$$

Hence

$$
\|f\|_{p, q, \alpha} \leq\left(\int_{0}^{1}(1-r)^{q \alpha-1}\left(\int_{0}^{r}(1-s)^{\beta-1} M_{p}\left(f^{(\beta)}, s\right) d s\right)^{q} d r\right)^{1 / q}=A .
$$

To estimate $A$ we consider first the case $0<q \leq 1$. Then

$$
\begin{aligned}
A^{q} & \leq \int_{0}^{1}(1-r)^{q \alpha-1} \int_{0}^{r}(1-s)^{\beta q-1} M_{p}^{q}\left(f^{(\beta)}, s\right) d s d r \\
& \leq \int_{0}^{1}(1-s)^{q \beta-1} M_{p}\left(f^{(\beta)}, s\right)\left(\int_{s}^{1}(1-r)^{q \alpha-1} d r\right) d s \\
& =(1 / q \alpha)\left\|f^{(\beta)}\right\|_{p, q, \alpha+\beta}^{q} .
\end{aligned}
$$

Assume now $q>1$. From(1.4) we have that $f^{(\beta)} \in H(p, 1, \alpha+\beta)$ and then integration by parts gives

$$
A^{q}=(1 / q \alpha) \int_{0}^{1}(1-r)^{q \alpha+\beta-1} M_{p}\left(f^{(\beta)}, r\right)\left(\int_{0}^{r}(1-s)^{\beta-1} M_{p}\left(f^{(\beta)}, s\right) d s\right)^{q-1} d r .
$$

Now writting $(1-r)^{q \alpha+\beta-1}=(1-r)^{\alpha+\beta-1 / q}(1-r)^{(q-1) \alpha-1 / q^{\prime}}$, Hölder's inequality gives

$$
A^{q} \leq C\left(\int_{0}^{1}(1-r)^{q(\alpha+\beta)-1} M_{p}^{q}\left(f^{(\beta)}, r\right) d r\right)^{1 / q} A^{q / q^{\prime}},
$$

which is the desired inequality.

For general value of $\beta$ we now argue as follows: If $f^{(\beta)} \in H(p, q, \alpha+\beta)$ and $0<\beta<1$ then $f^{(\beta+1)} \in H(p, q, \alpha+\beta+1)$ and now apply the previous case.

Let us finish this section with a theorem about duality that we shall use later. The reader is referred to [S1, F2, DRS, ACP, SW, Ma] for different duality resuls on several cases.

We denote by $H_{0}(p, q, \alpha)$ the closure of polynomials in $H(p, q, \alpha)$. It follows from standard techniques that the polynomials are dense in $H(p, q, \alpha)$ when $p, q<\infty$, that is $H_{0}(p, q, \alpha)=H(p, q, \alpha)$. Next theorem is due to T, M. Flett (see [F2, Theorem 2]). We present here a proof by using a pairing which is convinient for our purposes. 
Theorem B (Duality). Let $1 \leq p \leq \infty$ and $q \in\{1, \infty\}$. Then

$$
\left(H_{0}(p, q, \alpha)\right)^{*}=H\left(p^{\prime}, q^{\prime}, \alpha\right)
$$

under the pairing

$$
<f, g>_{\alpha}=\sum_{n=0}^{\infty} B(2 \alpha, n+1) a_{n} b_{n}
$$

where $B(a, b)=\int_{0}^{1}(1-r)^{a-1} r^{b-1} d r$.

Proof. Let $f(z)=\sum_{n=0}^{m} a_{n} z^{n}$ and $g(z)=\sum_{n=0}^{\infty} b_{n} z^{n}$. It is clear that

$$
<f, g>_{\alpha}=\frac{1}{4 \pi} \int_{0}^{1}\left(1-r^{2}\right)^{2 \alpha-1}\left(\int_{0}^{2 \pi} f\left(r e^{i \theta}\right) g\left(r e^{-i \theta}\right) d \theta\right) r d r
$$

Therefore using Hölder's inequality twice one gets

$$
\left|<f, g>_{\alpha}\right| \leq C \int_{0}^{1}(1-r)^{2 \alpha-1} M_{p}(f, r) M_{p^{\prime}}(g, r) r d r \leq C\|f\|_{p, q, \alpha}\|g\|_{p^{\prime}, q^{\prime}, \alpha} .
$$

Assume now $\phi \in\left(H_{0}(p, 1, \alpha)\right)^{*}$ and define $f(z)=\phi\left(K_{z}\right)$ where $K_{z}(w)=$ $\frac{1}{(1-z w)^{2 \alpha+1}}$.

Assume first $q=1$. Using duality one can write

$$
M_{p^{\prime}}(f, r)=\sup _{\|g\|_{p}=1}\left|\int_{0}^{2 \pi} f\left(r e^{i \theta}\right) g\left(e^{-i \theta}\right) \frac{d \theta}{2 \pi}\right|=\sup _{\|g\|_{p}=1}\left|\phi\left(h_{r}\right)\right|
$$

where $h_{r}(z)=\int_{0}^{2 \pi} \frac{g\left(e^{-i \theta}\right)}{\left(1-z r e^{i \theta}\right)^{2 \alpha+1}} \frac{d \theta}{2 \pi}=K_{r} * g(z)$.

Observe that since $M_{p}\left(h_{r}, s\right) \leq\|g\|_{p} M_{1}\left(K_{r}, s\right)$, a simple computation, using (1.2) and(1.3), gives $\left\|h_{r}\right\|_{p, 1, \alpha} \leq C_{\frac{1}{(1-r)^{\alpha}}}$. This and the previous equality imply that $f \in H\left(p^{\prime}, \infty, \alpha\right)$.

Assume now $q=\infty$. Let us denote by $f_{s}(z)=f(s z)$ for $0<s<1$. Using the previous case one has

$$
\left\|f_{s}\right\|_{p^{\prime}, 1, \alpha}=\sup _{\|g\|_{p, \infty, \alpha}=1}\left|<f_{s}, g>_{\alpha}\right|=\sup _{\|g\|_{p, \infty, \alpha}=1}\left|\phi\left(g_{s}\right)\right| .
$$

Observe now that $\left\|g_{s}\right\|_{p, \infty, \alpha} \leq\|g\|_{p, \infty, \alpha}$ for all $0<s<1$. Hence $\left\|f_{s}\right\|_{p^{\prime}, 1, \alpha} \leq C\|\phi\|$. Now apply Lebesgue convergence Theorem to get $f \in H\left(p^{\prime}, 1, \alpha\right)$.

\section{$\S 2$. THE SPACE $H(2, q, \alpha)$.}

A sequence space $S$ is called a solid if $\left(a_{n}\right) \in S$ and $\left|a_{n}^{\prime}\right| \leq\left|a_{n}\right|$ implies $\left(a_{n}\right) \in S$. It is clear, from Plancherel's theorem, that $H(2, q, \alpha)$ is a solid space. In fact, since $B(\beta, n) \approx n^{-\beta}$, we can identify $H(2,2, \alpha)$ with the space of sequences $\left(\lambda_{n}\right)$ such that $\left(n^{-\alpha} \lambda_{n}\right) \in l^{2}$. Our aim is to get similar identification for other values of $0<q<\infty$ (see [MP1, S1]). For such a purpose we shall need the following spaces. 
Definition 2.1. Let $0<p, q \leq \infty$. Denote by $I_{n}=\left\{k \in \mathbb{Z}: 2^{n-1} \leq k<2^{n}\right\}$ and $I_{0}=\{0\}$. The spaces $l(p, q)$ consist of sequences $\left(a_{n}\right) \in \mathbb{C}$ such that

$$
\left\|\left(a_{n}\right)\right\|_{p, q}=\left(\sum_{n}\left(\sum_{k \in I_{n}}\left|a_{k}\right|^{p}\right)^{q / p}\right)^{1 / q}<\infty
$$

(with the obvious modifications for the cases where $p$ or $q=\infty$.)

It is very elementary and well known that if $\alpha_{n} \geq 0$ and $\alpha>0$ then

$$
\sum_{n=0}^{\infty} \alpha_{n} r^{n}=O\left(\frac{1}{(1-r)^{\alpha}}\right) \text { if and only if } \sum_{k \in I_{n}} \alpha_{k}=O\left(2^{k \alpha}\right)
$$

As a simple consequence of (2.1) and Plancherel's theorem we can obtain, for any $\alpha>0$,

$$
H_{\alpha}^{2}=\left\{\left(\lambda_{n}\right): n^{-\alpha} \lambda_{n} \in l(2, \infty)\right\}
$$

To characterize $H(2, q, \infty)$ for other values of $q$ we shall use the following lemma.

Lemma 2.1. Let $0<q, \beta<\infty$ and $\alpha_{n} \geq 0$. Then

$$
\int_{0}^{1}(1-r)^{\beta q-1}\left(\sum_{n=0}^{\infty} \alpha_{n} r^{n}\right)^{q} d r \approx \sum_{n=0}^{\infty} 2^{-n \beta q}\left(\sum_{k \in I_{n}} \alpha_{k}\right)^{q}
$$

Proof.

$$
\begin{aligned}
\int_{0}^{1}(1-r)^{\beta q-1}\left(\sum_{n=0}^{\infty} \alpha_{n} r^{n}\right)^{q} d r & =\sum_{n=0}^{\infty} \int_{1-2^{-n}}^{1-2^{-(n+1)}}(1-r)^{\beta q-1}\left(\sum_{n=0}^{\infty} \alpha_{n} r^{n}\right)^{q} d r \\
& \geq C \sum_{n=0}^{\infty} 2^{-n(\beta q-1)} \int_{1-2^{-n}}^{1-2^{-(n+1)}}\left(\sum_{k \in I_{n}} \alpha_{k} r^{k}\right)^{q} d r \\
& \geq C \sum_{n=0}^{\infty} 2^{-n(\beta q-1)}\left(\sum_{k \in I_{n}} \alpha_{k}\right)^{q} \int_{1-2^{-n}}^{1-2^{-(n+1)}} r^{2^{n} q} d r \\
& \geq C \sum_{n=0}^{\infty} 2^{-n \beta q}\left(\sum_{k \in I_{n}} \alpha_{k}\right)^{q} .
\end{aligned}
$$

Let us now show the converse inequality. Assume first $0<q \leq 1$.

$$
\begin{aligned}
\int_{0}^{1}(1-r)^{\beta q-1}\left(\sum_{n=0}^{\infty} \alpha_{n} r^{n}\right)^{q} d r & \leq C \int_{0}^{1}(1-r)^{\beta q-1}\left(\sum_{n=0}^{\infty}\left(\sum_{k \in I_{n}} \alpha_{k}\right) r^{2^{n}-1}\right)^{q} d r \\
& \leq C \int_{0}^{1}(1-r)^{\beta q-1}\left(\sum_{n=0}^{\infty}\left(\sum_{k \in I_{n}} \alpha_{k}\right)^{q} r^{\left(2^{n}-1\right) q}\right) d r \\
& \leq C \sum_{n=0}^{\infty} \int_{0}^{1}(1-r)^{\beta q-1} r^{2^{n} q-1}\left(\sum_{k \in I_{n}} \alpha_{k}\right)^{q} d r \\
& \leq C \sum_{n=0}^{\infty} B\left(\beta q, 2^{n} q\right)\left(\sum_{k \in I_{n}} \alpha_{k}\right)^{q} \\
& \leq C \sum_{n=0}^{\infty} 2^{-n \beta q}\left(\sum_{k \in I_{n}} \alpha_{k}\right)^{q}
\end{aligned}
$$


Assume now $1<q<\infty$. Write

$$
\Phi\left(\lambda_{n}\right)=(1-r)^{\beta}\left(\sum_{n=0}^{\infty} n^{\beta} \lambda_{n} r^{n}\right) .
$$

Clearly (2.1) gives that $\Phi$ is a bounded operator from $l(1, \infty)$ to $L^{\infty}\left([0,1], \frac{d r}{1-r}\right)$ and the previous case $q=1$ shows that $\Phi$ is also bounded from $l(1,1)$ into $L^{1}\left([0,1], \frac{d r}{1-r}\right)$, then an interpolation argument shows

$$
\int_{0}^{1}(1-r)^{\beta q-1}\left(\sum_{n=0}^{\infty} \alpha_{n} r^{n}\right)^{q} d r \leq C \sum_{n=0}^{\infty} 2^{-n \beta q}\left(\sum_{k \in I_{n}} \alpha_{k}\right)^{q} .
$$

Theorem 2.1 (see $[\mathrm{S} 1, \mathrm{MP} 1])$. Let $0<q \leq \infty, \alpha>0$ and $f(z)=\sum_{n=1}^{\infty} a_{n} z^{n}$. Then

$$
f \in H(2, q, \alpha) \text { if and only if } n^{-\alpha} a_{n} \in l(2, q) .
$$

Proof. Use Plancherel's Theorem together with Lemma 2.1 or (2.1) according to $q<\infty$ or $q=\infty$ respectively.

Remark 2.1. Clearly one can formulate Theorem 2.1 by writing

$$
\|f\|_{2, q, \alpha} \approx\left(\sum_{n=0}^{\infty} 2^{-n q \alpha}\left\|\Delta_{n} f\right\|_{2}^{q}\right)^{1 / q}
$$

where $\Delta_{n} f(\theta)=\sum_{k \in I_{n}} a_{k} e^{i k \theta}$.

This can be extended to $1<p<\infty, 0<q<\infty$ and $\alpha>0$ (see [S1, MP2]) to get

$$
\|f\|_{p, q, \alpha} \approx\left(\sum_{n=0}^{\infty} 2^{-n q \alpha}\left\|\Delta_{n} f\right\|_{p}^{q}\right)^{1 / q}
$$

Using (2.3) one can easily obtain the following duality result for $1<p, q<\infty$ and $\alpha>0($ see $[\mathrm{S} 1])$

$$
(H(p, q, \alpha))^{*}=H\left(p^{\prime}, q^{\prime}, \alpha\right)
$$

under the pairing $<f, g>_{\alpha}=\sum_{n=0}^{\infty} 2^{-2 \alpha n} \int_{0}^{2 \pi} \Delta_{n} f(\theta) \Delta_{n} g(-\theta) \frac{d \theta}{2 \pi}$.

Given a sequence space $X$, we denote by $s(X)$ and $S(X)$ the largest solid subspace contained within $X$ and the smallest solid containing $X$ respectively.

It is not hard to show (see [BST, AS]) the following two characterizations:

$$
\begin{gathered}
s(X)=\left\{\lambda_{n}: a_{n} \lambda_{n} \in X \text { for every }\left(a_{n}\right) \in l^{\infty}\right\}=\left(l^{\infty}, X\right), \\
S(X)=\left\{\lambda_{n}: \text { there exists }\left(a_{n}\right) \in X \text { such that }\left|\lambda_{n}\right| \leq\left|a_{n}\right|\right\} .
\end{gathered}
$$

Let us give $S(H(p, q, \alpha))$ and $s(H(p, q, \alpha))$ in the cases which are easy to compute. Next results follows from either Kintchine's inequality the case $p<\infty$ or a result by Kisliakov (see $[\mathrm{Ki}]$ ) the case $p=\infty$. 
Lemma 2.2. Let $0<t \leq 2 \leq p<\infty, 0<q \leq \infty$ and $\alpha>0$. Then

$$
\begin{gathered}
S(H(\infty, q, \alpha))=H(2, q, \alpha), \\
S(H(p, q, \alpha))=H(2, q, \alpha), \\
s(H(t, q, \alpha))=H(2, q, \alpha) .
\end{gathered}
$$

Remark 2.2. We refer the reader to [BST, Theorem 1.8] and [MP2, Theorem 2.4] for a proof of (2.5), to [MP2, Theorem 2.5] for a proof of (2.6) and to [AS, Lemma $6]$ for a particular case of (2.7).

\section{$\S 3$. THE SPACE $H(p, q, \alpha)$ FOR $0<p \leq 1$.}

Our objective now is to extend (2.3) to values $0<p \leq 1$. This can be achieved by replacing the convolution with Dirichlet kernels $\sum_{k \in I_{n}} e^{i k \theta}$ by smooth kernels.

In $[\mathrm{JP}]$ the following functions $w_{n}$ were considered:

$$
w_{0}(z)=1+z, \quad w_{n}(z)=\sum_{k=2^{n-1}}^{2^{n+1}} \phi\left(\frac{k}{2^{n-1}}\right) z^{n}
$$

where $\phi(t)=w(t / 2)-w(t)$ and $w(t)$ is any infinitely differentiable function with $w(t)=1$ for $t \leq 1,0 \leq w(t) \leq 1$ for $1 \leq t \leq 2$ and $w(t)=0$ for $t \geq 2$.

For such functions they showed that for $0<p \leq 1$ and any analytic function $f$ one has

$$
\begin{gathered}
f=\sum_{n=0}^{\infty} f * w_{n}, \\
\left\|f * w_{n}\right\|_{p} \leq C\|f\|_{p} .
\end{gathered}
$$

Theorem 3.1. Let $0<p \leq 1,0<q, \alpha<\infty$ and $f$ an analytic function. Then

$$
\|f\|_{p, q, \alpha} \approx\left(\sum_{n=0}^{\infty} 2^{-n q \alpha}\left\|f * w_{n}\right\|_{p}^{q}\right)^{1 / q}
$$

Proof. Using (3.1) it easily follows

$$
M_{p}(f, r) \leq\left(\sum_{n=0}^{\infty} M_{p}^{p}\left(f * w_{n}, r\right)\right)^{1 / p} .
$$


Using now the fact (see [MP2, S2])

$$
M_{p}\left(f * w_{n}, r\right) \approx r^{2^{n}}\left\|f * w_{n}\right\|_{p},
$$

we can write

$$
\|f\|_{p, q, \alpha}^{q} \leq C \int_{0}^{1}(1-r)^{\alpha q-1}\left(\sum_{n=0}^{\infty}\left\|f * w_{n}\right\|_{p}^{p} r^{p 2^{n}}\right)^{q / p} d r .
$$

Applying now Lemma 2.1 we get

$$
\|f\|_{p, q, \alpha} \leq C\left(\sum_{n=0}^{\infty} 2^{-n q \alpha}\left\|f * w_{n}\right\|_{p}^{q}\right)^{1 / q} .
$$

For the converse inequality we use (3.2) and (3.4) to get

$$
\begin{aligned}
\int_{0}^{1}(1-r)^{q \alpha-1} M_{p}^{q}(f, r) d r & =\sum_{n=0}^{\infty} \int_{1-2^{-n}}^{1-2^{-(n+1)}}(1-r)^{q \alpha-1} M_{p}^{q}(f, r) d r \\
& \geq C \sum_{n=0}^{\infty} 2^{-n(q \alpha-1)} \int_{1-2^{-n}}^{1-2^{-(n+1)}} M_{p}^{q}(f, r) d r \\
& \geq C \sum_{n=0}^{\infty} 2^{-n(q \alpha-1)}\left\|f * w_{n}\right\|_{p}^{q} \int_{1-2^{-n}}^{1-2^{-(n+1)}} r^{2^{n} q} d r \\
& \geq C \sum_{n=1}^{\infty} 2^{-n q \alpha}\left\|f * w_{n}\right\|_{p}^{q} .
\end{aligned}
$$

Remark 3.1. A similar argument to the one used above, replacing $w_{n} * f$ by $\Delta_{n} f$, gives an alternative proof of (2.3) (the restriction on $1<p<\infty$ coming from the use of the boundedness of the Riesz transform).

Corollary 3.1. Let $0<p \leq 1$ and $0<q, \alpha<\infty$ and $f(z)=\sum_{n=0}^{\infty} a_{n} z^{n}$. Then

$$
\left\|n^{-\left(\alpha+\frac{1}{p}-1\right)} a_{n}\right\|_{\infty, q} \leq C\|f\|_{p, q, \alpha} .
$$

Proof. Note that if $\phi_{n}=w_{n-1}+w_{n}$ then $\left|\hat{\phi}_{n}(k)\right| \geq C$ for $k \in I_{n}$. Therefore

$$
\sup _{k \in I_{n}}\left|a_{k}\right| \leq \frac{1}{C}\left\|\phi_{n} * f\right\|_{1} .
$$

This together with (3.3) implies that for any $\beta$,

$$
\left\|n^{-\beta} a_{n}\right\|_{\infty, q} \leq C\|f\|_{1, q, \beta} .
$$

The proof is completed taking $\beta=\alpha+\frac{1}{p}-1$ and using that $\|f\|_{1, q, \alpha+\frac{1}{p}-1} \leq$ $\|f\|_{p, q, \alpha}$ which is given by (1.5). 
Corollary 3.2. Let $1 \leq p \leq 2 \leq s<\infty, 0<q<\infty, 1<t<\infty$ and $0<\alpha, \beta<\infty$. If $f(z)=\sum_{n=0}^{\infty} a_{n} z^{n}$ then

$$
\begin{gathered}
\left\|n^{-\alpha} a_{n}\right\|_{p^{\prime}, q} \leq C\|f\|_{p, q, \alpha}, \\
\|f\|_{s, t, \beta} \leq C\left\|n^{-\beta} a_{n}\right\|_{s^{\prime}, t} .
\end{gathered}
$$

Proof. Using Corollary 3.1 and Theorem 2.2 we have

$$
\begin{gathered}
H(1, q, \alpha) \subset\left\{a_{n}: n^{-\alpha} a_{n} \in l(\infty, q)\right\}, \\
H(2, q, \alpha)=\left\{a_{n}: n^{-\alpha} a_{n} \in l(2, q)\right\} .
\end{gathered}
$$

Hence an interpolation argument gives

$$
H(p, q, \alpha) \subset\left\{a_{n}: n^{-\alpha} a_{n} \in l\left(p^{\prime}, q\right)\right\} .
$$

To get (3.5) we apply duality. Since $1<s^{\prime}, t^{\prime}<\infty$ applying (2.4) we get

$$
<f, g>_{\beta}=\sum_{n=0}^{\infty} 2^{-2 \beta n} \sum_{k \in I_{n}} a_{k} b_{k} .
$$

Then (3.4) easily gives

$$
\begin{aligned}
\|f\|_{s, t, \beta} & =\sup _{\|g\|_{s^{\prime}, t^{\prime}, \beta} \leq 1}\left|\sum_{n=0}^{\infty} 2^{-2 \beta n} \sum_{k \in I_{n}} a_{k} b_{k}\right| \\
& \leq \sup _{\|g\|_{s^{\prime}, t^{\prime}, \beta} \leq 1}\left\|\left(n^{-\beta} a_{n}\right)\right\|_{s^{\prime}, t}\left\|\left(n^{-\beta} b_{n}\right)\right\|_{s, t^{\prime}} \\
& \leq C\left\|\left(n^{-\beta} a_{n}\right)\right\|_{s^{\prime}, t} .
\end{aligned}
$$

Next we shall show that the study of multipliers and, in general, of operators acting on $H(p, q, \alpha)$ for $0<p, q \leq 1$ relies upon the case $H\left(1,1, \alpha+\frac{1}{p}-1\right)$. The reader is referred to [B1] for a more general formulation of the following result and its applications.

Theorem 3.2. Let $\alpha>0,0<p, q \leq 1$ and $E$ be a Banach space. Let $T$ be a linear map from the space of polynomials into $E$. Then the following are equivalent

(i) $T$ extends to a bounded operator from $H(p, q, \alpha)$ into $E$.

(ii) $\left\|T\left(K_{w}\right)\right\|_{E}=O\left(\frac{1}{(1-|w|)^{\alpha}}\right) \quad$ where $K_{w}(z)=\frac{1}{(1-z \bar{w})^{2 \alpha+\frac{1}{p}}}$

(iii) $T$ extends to a bounded operator from $H\left(1,1, \alpha+\frac{1}{p}-1\right)$ into $E$.

Proof. $(a) \Rightarrow(b)$. It follows from estimating $\left\|K_{w}\right\|_{p, q, \alpha}$, since $\left\|T\left(K_{w}\right)\right\|_{E} \leq C\left\|K_{w}\right\|_{p, q, \alpha}$.

Using (1.2) and (1.3) we have

$$
\left(\int_{0}^{1}(1-r)^{\alpha q-1} M_{p}^{q}\left(K_{w}, r\right) d r\right)^{1 / q} \leq C\left(\int_{0}^{1} \frac{(1-r)^{\alpha q-1}}{(1-|w| r)^{2 \alpha q}} d r\right)^{1 / q} \leq C \frac{1}{(1-|w|)^{\alpha}} .
$$


$(b) \Rightarrow(c)$. Since the polynomials are dense in $H\left(1,1, \alpha+\frac{1}{p}-1\right)$ then it suffices to prove $\|T(f)\|_{E} \leq C\|f\|_{1,1, \alpha+\frac{1}{p}-1}$ for all polynomials $f$.

It is immediate to show that if $\beta>0$ and $f$ is a polynomial then

$$
f(z)=\int_{0}^{1} \int_{0}^{2 \pi}(1-r)^{\beta-1} \frac{f\left(r e^{i \theta}\right)}{\left(1-z r e^{-i \theta}\right)^{\beta+1}} d r \frac{d \theta}{2 \pi} .
$$

Take $\beta=2 \alpha+\frac{1}{p}-1$ and a polynomial $f$, then we have

$$
f(z)=\int_{0}^{1} \int_{0}^{2 \pi}(1-r)^{\beta-1} f\left(r e^{i \theta}\right) K_{r e^{-i \theta}}(z) d r \frac{d \theta}{2 \pi} .
$$

Then, by linearity

$$
T(f)=\int_{0}^{1} \int_{0}^{2 \pi}(1-r)^{\beta-1} f\left(r e^{i \theta}\right) T\left(K_{r e^{-i \theta}}\right) d r \frac{d \theta}{2 \pi} .
$$

Therefore

$$
\begin{aligned}
\|T(f)\|_{E} & \leq \int_{0}^{1} \int_{0}^{2 \pi}(1-r)^{\beta-1}\left|f\left(r e^{i \theta}\right)\right|\left\|T\left(K_{r e^{-i \theta}}\right)\right\|_{E} d r \frac{d \theta}{2 \pi} \\
& \leq C \int_{0}^{1}(1-r)^{\beta-1} \frac{M_{1}(f, r)}{(1-r)^{\alpha}} d r=C\|f\|_{1,1, \alpha+\frac{1}{p}-1}
\end{aligned}
$$

$(c) \Rightarrow(a)$. It follows from (1.5).

Remark 3.2. Note that the previous result essentially shows that the Banach envelope of $H(p, q, \alpha)$ for $0<p, q \leq 1$ is $H\left(1,1, \alpha+\frac{1}{p}-1\right.$ ) (see [DRS, Sh] for particular cases).

\section{§4. Multipliers $\left(H(p, q, \alpha), l^{s}\right)$.}

There is a general procedure to deal with multipliers from spaces of analytic functions into $l^{p}$ spaces (see [BST, AS]) which consists of identifying the smallest solid containing the space. This approach can be applied for values $2 \leq p \leq \infty$.

Lemma 4.1 (see [AS, Lemma 3]). Let $X$ be a sequence space and $S$ be a solid. Then

$$
\begin{aligned}
& (X, S)=(S(X), S), \\
& (S, X)=(S, s(X)) .
\end{aligned}
$$

Lemma 4.2 (see $[\mathrm{K}])$. Let $0<p_{1}, q_{1}, p_{2}, q_{2} \leq \infty$. If we write $\frac{1}{p}=\frac{1}{\min \left(p_{1}, p_{2}\right)}-\frac{1}{p_{1}}$ and $\frac{1}{q}=\frac{1}{\min \left(q_{1}, q_{2}\right)}-\frac{1}{q_{1}}$ then

$$
\left(l\left(p_{1}, q_{1}\right), l\left(p_{2}, q_{2}\right)\right)=l(p, q)
$$

As a simple consequence of (4.1), Lemmas 2.2 and 4.2 and Theorem 2.1, we can state the following 
Theorem 4.1. Let $2 \leq p \leq \infty, 0<q \leq \infty$ and $0<\alpha<\infty$. If $\frac{1}{r}=\frac{1}{\min (s, 2)}-\frac{1}{2}$ and $\frac{1}{t}=\frac{1}{\min (s, q)}-\frac{1}{q}$ then

$$
\left(H(p, q, \alpha), l^{s}\right)=\left\{\lambda_{n}: n^{\alpha} \lambda_{n} \in l(r, t)\right\} .
$$

Now we shall try to study the case $H(p, q, \alpha)$ for $p \leq 1$. Our main tool will be the Theorem 3.2.

Theorem 4.2. Let $0<p, q \leq 1 \leq s \leq \infty$

$$
\left(H(p, q, \alpha), l^{s}\right)=\left\{\lambda_{n}: n^{\alpha+\frac{1}{p}-1} \lambda_{n} \in l(s, \infty)\right\} .
$$

Proof. Apply Theorem 3.2 and observe that condition (ii) says that $\left(\lambda_{n}\right)$ belongs to $\left(H(p, q, \alpha), l^{s}\right)$ if and only if

$$
\sum_{n=0}^{\infty}\left(\left|\mu_{n}\right|\left|\lambda_{n}\right||w|^{n}\right)^{s}=O\left(1 /(1-|w|)^{\alpha s}\right)
$$

where $\left(\mu_{n}\right)$ are the Taylor coefficients of $1 /(1-z)^{2 \alpha+1 / p}$.

Therefore, estimating $\mu_{n} \approx n^{2 \alpha+1 / p-1}$ and using (2.1) we get $n^{\alpha+\frac{1}{p}-1} \lambda_{n} \in$ $l(s, \infty)$.

Remark 4.1. The case $p=q=1$ of the previous result was already known (see [DS1, Theorem 2]), and then, the equivalence between (i) and (iii) in Theorem 3.2 might have been used, but we have decided to include here this simple direct proof.

Theorem 4.3. Let $0<p \leq 1 \leq q \leq s$ and $0<\alpha<\infty$. If $\frac{1}{r}=\frac{1}{\min \left(\frac{s}{s-q}, q\right)}+\frac{q}{s}-1$ then

$$
\left(H(p, q, \alpha), l^{s}\right)=\left\{\lambda_{n}: n^{\alpha+\frac{1}{p}-1} \lambda_{n} \in l(r, \infty)\right\} .
$$

Proof. Let us start with the case $s=q$. Then also $r=q$. Assume first that $\left(\lambda_{n}\right) \in\left(H(p, q, \alpha), l^{s}\right)$. Denoting by $V_{n}$ the de la Vallé Poussin kernel, we consider

$$
\phi_{n}(t)=V_{2^{n}}\left(t+2^{n}\right)
$$

Since $\left\|V_{n}\right\|_{1} \leq 3$ for all $n \in \mathbb{N}$ then it follows that $M_{1}\left(\phi_{n}, r\right) \leq C r^{2^{n}}$ and therefore $\|\phi\|_{1, q, \beta} \leq C 2^{-n \beta}$ for any $\beta>0$.

On the other hand $V_{n}$ is a polynomial of degree $2 \mathrm{n}+1$ having $\hat{V}_{n}(j)=1$ for $|j| \leq n+1$ and then the assumption on $\left(\lambda_{n}\right)$ and (1.5) imply

$$
\begin{aligned}
\left(\sum_{k \in I_{n}}\left|\lambda_{n}\right|^{s}\right)^{1 / s} & \leq\left(\sum_{k \in I_{n}}\left|\hat{\phi}_{n}(k) \lambda_{k}\right|^{s}\right)^{1 / s} \leq C\|\phi\|_{p, q, \alpha} \\
& \leq C\|\phi\|_{1, q, \alpha+1 / p-1} \leq C 2^{-n(\alpha+1 / p-1)} .
\end{aligned}
$$


Assume now that $n^{\alpha+\frac{1}{p}-1} \lambda_{n} \in l(q, \infty)$ and use Corollary 3.1 to have

$$
\begin{aligned}
\sum_{n=0}^{\infty}\left|\lambda_{n}\right|^{q}\left|a_{n}\right|^{q} & \leq \sum_{n=0}^{\infty}\left(\sum_{k \in I_{n}}\left|\lambda_{n}\right|^{q}\left|a_{n}\right|^{q}\right) \\
& \leq \sum_{n=1}^{\infty}\left(\sum_{k \in I_{n}} n^{\left(\alpha+\frac{1}{p}-1\right) q}\left|\lambda_{n}\right|^{q}\right)\left(\sup _{k \in I_{n}} n^{-\left(\alpha+\frac{1}{p}-1\right) q}\left|a_{n}\right|^{q}\right) \\
& \leq C\left\|n^{\alpha+\frac{1}{p}-1} \lambda_{n}\right\|_{q, \infty}^{q}\left\|n^{-\left(\alpha+\frac{1}{p}-1\right)} a_{n}\right\|_{\infty, q}^{q} \leq C\|f\|_{p, q, \alpha}^{q} .
\end{aligned}
$$

Let us now show that the case $q<s$ follows from the previous one. Using duality one has that if $\frac{1}{t}=1-\frac{q}{s}$ then

$\left(\lambda_{n}\right) \in\left(H(p, q, \alpha), l^{s}\right)^{s}$ if and only if $\left(\lambda_{n} \beta_{n}\right) \in\left(H(p, q, \alpha), l^{q}\right)$ for all $\left(\beta_{n}\right) \in l^{t}$.

From the previous case this means $\left(n^{\alpha+\frac{1}{p}-1} \lambda_{n} \beta_{n}\right) \in l(q, \infty)$ for all $\left(\beta_{n}\right) \in l^{t}$, that is $n^{\alpha+\frac{1}{p}-1} \lambda_{n} \in(l(t, t), l(q, \infty))$. Then the proof is completed by invoking Lemma 4.2.

Remark 4.2. For $0<p \leq 1$ and $q=\infty$ the multipliers can be characterized for all $0<s \leq \infty$. The reader is referred to [M, Theorem 1] for a proof of the following result

$$
\left(H_{\alpha}^{p}, l^{s}\right)=\left\{\lambda_{n}: n^{\alpha+\frac{1}{p}-1} \lambda_{n} \in l^{s}\right\} .
$$

Let us conclude with a partial result for the values $1 \leq p \leq 2$.

Theorem 4.4. Let $1 \leq p \leq 2$. Let $0<q<\infty$ and $s \leq \min \left(p^{\prime}, q\right)$. Put $\frac{1}{p}+\frac{1}{p^{\prime}}=1$, $\frac{1}{r}=\frac{1}{s}-\frac{1}{p^{\prime}}$ and $\frac{1}{t}=\frac{1}{s}-\frac{1}{q}$.

$$
\text { If }\left(n^{\alpha} \lambda_{n}\right) \in l(r, t) \text { then }\left(\lambda_{n}\right) \in\left(H(p, q, \alpha), l^{s}\right) \text {. }
$$

Proof. Using (3.4) and Hölder's inequality for $\frac{p^{\prime}}{s}$ and $\frac{q}{s}$ consecutively we have

$$
\begin{aligned}
\sum_{n=0}^{\infty}\left|\lambda_{n}\right|^{s}\left|a_{n}\right|^{s} & \leq \sum_{n=1}^{\infty}\left(\sum_{k \in I_{n}}\left|\lambda_{n}\right|^{s}\left|a_{n}\right|^{s}\right) \\
& \sum_{n=1}^{\infty}\left(\sum_{k \in I_{n}} n^{-\alpha p^{\prime}}\left|a_{n}\right|^{p^{\prime}}\right)^{s / p^{\prime}}\left(\sum_{k \in I_{n}} n^{\alpha r}\left|\lambda_{n}\right|^{r}\right)^{s / r} \\
& \leq C\left\|\left(n^{-\alpha} a_{n}\right)\right\|_{p^{\prime}, q}^{s}\left(\sum_{n=1}^{\infty}\left(\sum_{k \in I_{n}} n^{\alpha r}\left|\lambda_{n}\right|^{r}\right)^{t / r}\right)^{s / t} \\
& \leq C\|f\|_{p, q, \alpha}^{s}\left\|n^{\alpha} \lambda_{n}\right\|_{r, t}^{s} \cdot \square
\end{aligned}
$$

§5. Multipliers $(H(p, q, \alpha), H(s, t, \beta))$.

Lemma 5.1. Let $0<p, q<\infty$. Let $f(z)=\sum_{n=0}^{\infty} a_{n} z^{n}$. Let us denote by $f_{x}(z)=\sum_{n=0}^{\infty} r_{n}(x) a_{n} z^{n}$ where $r_{n}$ stand for the Rademacher functions. Then

$$
M_{2}^{q}(f, r) \approx \int_{0}^{1} M_{p}^{q}\left(f_{x}, r\right) d x
$$


Proof. For $p \leq q$ we have

$$
\left(\int_{0}^{1} M_{p}^{p}\left(f_{x}, r\right) d x\right)^{1 / p} \leq\left(\int_{0}^{1} M_{p}^{q}\left(f_{x}, r\right) d x\right)^{1 / q} \leq\left(\int_{0}^{1} M_{q}^{q}\left(f_{x}, r\right) d x\right)^{1 / q} .
$$

For $q \leq p$ we have

$$
\left(\int_{0}^{1} M_{q}^{q}\left(f_{x}, r\right) d x\right)^{1 / q} \leq\left(\int_{0}^{1} M_{p}^{q}\left(f_{x}, r\right) d x\right)^{1 / q} \leq\left(\int_{0}^{1} M_{p}^{p}\left(f_{x}, r\right) d x\right)^{1 / p} .
$$

Then the proof follows form these estimates together with Kintchine's inequality which says

$$
\left(\int_{0}^{1} M_{q}^{q}\left(f_{x}, r\right) d x\right)^{1 / q} \approx M_{2}(f, r) \approx\left(\int_{0}^{1} M_{p}^{p}\left(f_{x}, r\right) d x\right)^{1 / p} .
$$

Theorem 5.1. Let $0<s \leq 2 \leq p<\infty, 0<q, t<\infty$ and $\alpha, \beta>0$. Let $1 / r=1 / \min (q, t)-1 / q$. Then

$$
(H(p, q, \alpha), H(s, t, \beta))=\left\{\left(\lambda_{n}\right): n^{\alpha-\beta} \lambda_{n} \in l(\infty, r)\right\} .
$$

Proof. Using Theorem 2.1 and Lemma 4.2 we have

$$
(H(2, q, \alpha), H(2, t, \beta))=\left\{\left(\lambda_{n}\right): n^{\alpha-\beta} \lambda_{n} \in l(\infty, r)\right\} .
$$

We shall show that

$$
(H(p, q, \alpha), H(s, t, \beta))=(H(2, q, \alpha), H(2, t, \beta)) .
$$

It is immediate that $((H(2, q, \alpha), H(2, t, \beta)) \subset((H(p, q, \alpha), H(s, t, \beta))$.

Take now $\lambda_{n} \in\left((H(p, q, \alpha), H(s, t, \beta))\right.$ and $f(z)=\sum_{n=0}^{\infty} a_{n} z^{n} \in H(p, q, \alpha)$. Denote by $(f * \lambda)_{x}(z)=\sum_{n=0}^{\infty} r_{n}(x) a_{n} \lambda_{n} z^{n}$.

In the case $t<\infty$ an application of Lemma 5.1 and Fubini's Theorem give

$$
\begin{aligned}
\int_{0}^{1}(1-r)^{\beta t-1} M_{2}^{t}(f * \lambda, r) d r & \leq C \int_{0}^{1}(1-r)^{\beta t-1}\left(\int_{0}^{1} M_{s}^{t}\left((f * \lambda)_{x}, r\right) d x\right) d r \\
& \leq C \int_{0}^{1}\left(\int_{0}^{1}(1-r)^{\beta t-1} M_{s}^{t}\left(f_{x} * \lambda, r\right) d r\right) d x \\
& \leq C \int_{0}^{1}\left(\int_{0}^{1}(1-r)^{\alpha q-1} M_{p}^{q}\left(f_{x}, r\right) d r\right)^{t / q} d x=A
\end{aligned}
$$

Now if $t \leq q$ then apply Jensen's inequality, Fubini and use Lemma 5.1 again, to get

$$
\begin{aligned}
A & \leq C\left(\int_{0}^{1}(1-r)^{\alpha q-1}\left(\int_{0}^{1} M_{p}^{q}\left(f_{x}, r\right) d x\right) d r\right)^{t / q} \\
& \leq C\left(\int_{0}^{1}(1-r)^{\alpha q-1} M_{2}^{q}(f, r) d r\right)^{t / q} .
\end{aligned}
$$


If $t>q$ put $r=\frac{t}{q}$ and apply duality and Lemma 5.1 to get

$$
\begin{aligned}
A & \leq C \sup _{\|h\|_{r^{\prime}=1}}\left(\int_{0}^{1}\left(\int_{0}^{1}(1-r)^{\alpha q-1} M_{p}^{q}\left(f_{x}, r\right) d r\right) h(x) d x\right)^{t / q} \\
& \leq C\left(\int_{0}^{1}(1-r)^{\alpha q-1}\left(\int_{0}^{1} M_{p}^{t}\left(f_{x}, r\right) d x\right)^{q / t} d r\right)^{t / q} \\
& \leq C\left(\int_{0}^{1}(1-r)^{\alpha q-1} M_{2}^{q}(f, r) d r\right)^{t / q} \cdot \square
\end{aligned}
$$

Theorem 5.2. Let $1<s \leq 2 \leq p \leq \infty, 1<t \leq \infty$ and $\alpha, \beta>0$. Then

$$
\left(H_{\alpha}^{p}, H(s, t, \beta)\right)=\left\{\left(\lambda_{n}\right): n^{\alpha-\beta} \lambda_{n} \in l(\infty, t)\right\} .
$$

Proof. Arguing as in the previous theorem we only need to show

$$
\left(H_{\alpha}^{p}, H(s, t, \beta)\right) \subset\left(H_{\alpha}^{2}, H(2, t, \beta)\right) .
$$

Observe that $(X, Y) \subset\left(Y^{*}, X^{*}\right)$ then Theorem B or (2.4) in Remark 2.1 imply $(H(p, \infty, \alpha), H(s, t, \beta)) \subset\left(H_{0}(p, \infty, \alpha), H_{0}(s, t, \beta)\right) \subset\left(H\left(s^{\prime}, t^{\prime}, \beta\right), H\left(p^{\prime}, 1, \alpha\right)\right)$.

Now apply Theorem 5.1 and duality again to get

$$
\left(H\left(s^{\prime}, t^{\prime}, \beta\right), H\left(p^{\prime}, 1, \alpha\right)\right)=\left(H\left(2, t^{\prime}, \beta\right), H(2,1, \alpha)\right) \subset(H(2, \infty, \alpha), H(2, t, \beta)) .
$$

The proof is completed by combining both sequences of embeddings.

Remark 5.1. Theorems 5.1 and 5.2 have a natural extension to weights more general than power weights $w(t)=t^{\alpha}($ see $[\mathrm{B} 2])$.

Lemma 5.2. Let $0<p, q \leq \infty$ and $0<\alpha, \beta<\infty$. Let $f$ be an analytic function on the disc and $f_{r}(z)=f(r z)$. Then

$$
f \in H_{\alpha+\beta}^{p} \quad \text { if and only if } \quad\left\|f_{r}\right\|_{p, q, \alpha}=O\left(\frac{1}{(1-r)^{\beta}}\right) .
$$

Proof. Let us assume $q<\infty$ (since $q=\infty$ is obvious) and $M_{p}(f, s)=O\left(\frac{1}{(1-s)^{\alpha+\beta}}\right)$. Then it follows from (1.3) that

$$
\int_{0}^{1}(1-s)^{q \alpha-1} M_{p}^{q}\left(f_{r}, s\right) d s \leq C \int_{0}^{1} \frac{(1-s)^{q \alpha-1}}{(1-r s)^{(\alpha+\beta) q}} d s \leq \frac{C}{(1-r)^{q \beta}} .
$$

Conversely observe that

$$
(1-s)^{\alpha} M_{p}\left(f_{r}, s\right) \leq\left(\int_{s}^{1}(1-t)^{\alpha q-1} M_{p}^{q}\left(f_{r}, t\right) d t\right)^{1 / q} \leq\left\|f_{r}\right\|_{p, q, \alpha} .
$$

Therefore if $\left\|f_{r}\right\|_{p, q, \alpha}=O\left(\frac{1}{(1-r)^{\beta}}\right)$ then $M_{p}(f, r s) \leq \frac{C}{(1-s)^{\alpha}(1-r)^{\beta}}$ which gives $f \in$ $H_{\alpha+\beta}^{p}$. 
Theorem 5.3. Let $0<p, q \leq 1,1 \leq s, t \leq \infty$ and $\alpha, \beta>0$. Let $\gamma=\alpha+1 / p-1$. Then

$$
(H(p, q, \alpha), H(s, t, \beta))=\left\{\left(\lambda_{n}\right): \lambda^{(\gamma)} \in H_{\beta}^{s}\right\} .
$$

Proof. From Theorem 3.2 the condition for $\lambda$ to be a multiplier is

$$
\left\|\lambda * K_{w}\right\|_{s, t, \beta}=O\left(\frac{1}{(1-|w|)^{\alpha}}\right)
$$

where $K_{w}(z)=1 /(1-z \bar{w})^{2 \alpha+1 / p}$. In other words, writing $\delta=2 \alpha+1 / p-1$ and $w=r$

$$
\left\|\lambda_{r}^{(\delta)}\right\|_{s, t, \beta}=O\left(\frac{1}{(1-r)^{\alpha}}\right) .
$$

Hence Lemma 5.2 implies that $\lambda^{(\delta)} \in H_{\alpha+\beta}^{s}$ and then (1.12) gives $\lambda^{(\gamma)} \in H_{\beta}^{s}$.

Theorem 5.4. Let $0<p \leq 1,1 \leq s, q \leq \infty$ and $\alpha, \beta>0$. Let $\gamma=\alpha+1 / p-1$. Then

$$
(H(p, q, \alpha), H(s, q, \beta))=\left\{\left(\lambda_{n}\right): \lambda^{(\gamma)} \in H_{\beta}^{s}\right\} .
$$

Proof. Assume $\left(\lambda_{n}\right) \in(H(p, q, \alpha), H(s, q, \beta))$ and fix $0<r<1$. Therefore

$$
\left\|\sum_{n=0}^{\infty} \frac{\Gamma(2 \gamma+n+1)}{\Gamma(2 \gamma) n !} \lambda_{n} r^{n} z^{n}\right\|_{s, q, \beta} \leq C\left\|K_{\gamma, r}\right\|_{p, q, \alpha}
$$

where $K_{\gamma, r}(z)=\frac{1}{(1-r z)^{2 \gamma+1}}$.

Since $\left\|K_{\gamma, r}\right\|_{p, q, \alpha} \leq \frac{C}{(1-r)^{\gamma}}$ then $\left\|\lambda_{r}^{(2 \gamma)}\right\|_{s, q, \beta} \leq \frac{C}{(1-r)^{\gamma}}$. Now Lemma 5.2 and (1.12) imply that $\lambda^{(\gamma)} \in H_{\beta}^{s}$.

For the converse first note that since $H(p, q, \alpha) \subset H(1, q, \gamma)$ then it suffices to show that if $\lambda^{\gamma} \in H_{\beta}^{s}$ then $\lambda \in(H(1, q, \gamma), H(s, q, \beta))$.

Assume first that $\gamma \geq \frac{1}{2}$ and $\lambda^{(\gamma)} \in H_{\beta}^{s}$. From (1.12) we have $\lambda^{(2 \gamma)} \in H_{\gamma+\beta}^{s}$.

To show that $\left(\lambda_{n}\right) \in(H(p, q, \alpha), H(s, q, \beta))$ is equivalent, by duality (see Theorem $\mathrm{B}$ and (2.4), to show that

$$
\left|\sum_{n=0}^{\infty} B(2 \beta, n+1) \lambda_{n} a_{n} b_{n}\right| \leq C\|f\|_{1, q, \gamma}\|g\|_{s^{\prime}, q^{\prime}, \beta},
$$

for all $f(z)=\sum_{n=0}^{\infty} a_{n} z^{n} \in H(1, q, \gamma)$ and $g(z)=\sum_{n=0}^{\infty} b_{n} z^{n} \in H_{0}\left(s^{\prime}, q^{\prime}, \beta\right)$.

On the other hand given $f$ and $g$ as above, (1.7) in Lemma $\mathrm{C}$ implies that $\sum_{n=0}^{\infty} a_{n} b_{n} z^{n} \in H_{0}\left(s^{\prime}, 1, \gamma+\beta\right)$, and duality again gives

$$
\begin{aligned}
\left|\sum_{n=0}^{\infty} B(2 \beta, n+1) \lambda_{n} a_{n} b_{n}\right|= & \left|\sum_{n=0}^{\infty} B(2 \beta+2 \gamma, n+1) \mu_{n} a_{n} b_{n}\right| \\
& \leq C\|h\|_{s, \infty, \gamma+\beta}\|f * g\|_{s^{\prime}, 1, \gamma+\beta}
\end{aligned}
$$


where $\mu_{n}=\frac{B(2 \beta, n+1)}{B(2 \beta+2 \gamma, n+1)} \lambda_{n}$ and $h(z)=\sum_{n=0}^{\infty} \mu_{n} z^{n}$.

Hence the proof of (5.1) will be finished, using (1.7), by showing $h \in H_{\gamma+\beta}^{s}$.

Now observe that denoting by $\delta_{n}=\frac{B(2 \beta, n+1)}{B(2(\beta+\gamma), n+1)}$, a simple computation, under the assumption $\gamma \geq \frac{1}{2}$, shows that $\delta_{n+1}+\delta_{n-1} \geq 2 \delta_{n}$. This allows us to consider $K(t)=\sum_{-\infty}^{\infty} \delta_{n} e^{i n t}$ which from the convexity condition is integrable (see [Ka, page $22])$. Therefore since $h\left(r e^{i \theta}\right)=K * \lambda\left(r e^{i \theta}\right)$ we get $\|h\|_{s, \infty, \gamma+\beta} \leq C\left\|\lambda^{(2 \gamma)}\right\|_{s, \infty, \gamma+\beta}$.

The case $\gamma<\frac{1}{2}$ follows from the previous case by observing that (1.12) gives that $\lambda^{(\gamma)} \in H_{\beta}^{s}$ is equivalent to $\lambda^{(\gamma+1)} \in H_{\beta+1}^{s}$ and that Theorem A gives that

$$
(H(1, q, \gamma), H(s, q, \beta))=(H(1, q, \gamma+1), H(s, q, \beta+1)) .
$$

Let us finisish the section with a partial result for $1 \leq p \leq 2$.

Theorem 5.5. Let $1 \leq p \leq 2 \leq s<\infty$. Let $0<q<\infty, 1<t<\infty$ and $0<\alpha, \beta<\infty$. Put $\frac{1}{r}=\frac{1}{p}-\frac{1}{s}$ and $\frac{1}{u}=\frac{1}{\min \{q, t\}}-\frac{1}{q}$.

$$
\text { If }\left(n^{\alpha-\beta} \lambda_{n}\right) \in l(r, u) \text { then }\left(\lambda_{n}\right) \in(H(p, q, \alpha), H(s, t, \beta)) \text {. }
$$

Proof. The proof follows by combining Corollary 3.2 and Lemma 4.2.

If $f(z)=\sum_{n=0}^{\infty} a_{n} z^{n}$ we can write

$$
\begin{aligned}
\left\|\sum_{n=0}^{\infty} \lambda_{n} a_{n} z^{n}\right\|_{s, t, \beta} & \leq\left\|\left(n^{-\beta} \lambda_{n} a_{n}\right)\right\|_{s^{\prime}, t} \\
& \leq\left\|\left(n^{\alpha-\beta} \lambda_{n}\right)\right\|_{r, u}\left\|\left(n^{-\alpha} a_{n}\right)\right\|_{p^{\prime}, q} \\
& \leq C\left\|\left(n^{\alpha-\beta} \lambda_{n}\right)\right\|_{r, u}\|f\|_{p, q, \alpha} . q e d
\end{aligned}
$$

§6. Applications to multipliers on Bergman and Hardy sPaCES.

Since $B_{p}=H(p, p, 1 / p)$ Theorems 5.1 and 5.2 give

Corollary 6.1 (see [W, Theorems 7 and 11]). Let $\alpha>0,1<p \leq 2 \leq q<\infty$ and $\frac{1}{r}=\frac{1}{q}-\frac{1}{p}$. Then

$$
\begin{gathered}
\left(B_{q}, B_{p}\right)=\left\{\lambda_{n}: n^{-\frac{1}{r}} \lambda_{n} \in l(r, \infty)\right\}, \\
\left(H_{\alpha}^{\infty}, B_{p}\right)=\left\{\lambda_{n}: n^{\alpha-\frac{1}{p}} \lambda_{n} \in l(p, \infty)\right\} .
\end{gathered}
$$

Remark 6.1. Assume $\lambda_{n} \in(X, Y)$ can be written as $\lambda_{n}=\mu_{n} \nu_{n}$ where $\mu_{n} \in(X, S)$ and $\nu_{n} \in(S, Y)$ for some solid space $S$, then it follows from (4.1) and (4.2) that $(X, Y)=(S(X), s(Y))$. Therefore an alternative proof of Corollary 6.1 consists of showing that $\lambda_{n} \in\left(B_{q}, B_{p}\right)$ can be written as $\lambda_{n}=\mu_{n} \nu_{n}$ where $\mu_{n} \in\left(B_{q}, l^{2}\right)$ and $\nu_{n} \in\left(l^{2}, B_{p}\right)$ (see [W, Proposition 1]).

Let us recall that an analytic function is said to be a Bloch function if

$$
\left|f^{\prime}(z)\right|=O(1 /(1-|z|) .
$$

Hence we have for any $\alpha>0$

$$
\text { Bloch }=\left\{f: f^{(\alpha)} \in H(\infty, \infty, \alpha)\right\} .
$$

With this notation Theorems 5.3 and 5.4 give the following 
Corollary 6.2. Let $1 \leq p, q \leq \infty, 1 \leq t \leq \infty$ and $\beta>\alpha>0$.

$$
\begin{gathered}
(H(1,1, \alpha), H(\infty, t, \alpha))=\text { Bloch, } \\
(H(1, q, \alpha), H(\infty, q, \alpha))=\text { Bloch, } \\
\left(H_{\alpha}^{1}, H_{\beta}^{p}\right)=H_{\beta-\alpha}^{p} .
\end{gathered}
$$

To get our next applications let us denote by $J_{p, q}$ the space of analytic functions $f$ such that $f^{\prime} \in H(p, q, 1)$. With this notation Bloch corresponds to $J_{\infty, \infty}$, and Lemma D allows us to write the following embeddings:

$$
J_{1,1} \subset H^{1} \subset J_{1,2}
$$

Note that for "nice" analytic functions $f$ and $g$

$$
\int_{0}^{2 \pi} f\left(e^{i \theta}\right) g\left(e^{-i \theta}\right) \frac{d \theta}{2 \pi}=\frac{1}{2} \int_{0}^{1} \int_{0}^{2 \pi}\left(1-r^{2}\right) f^{\prime}\left(r e^{i \theta}\right) g^{\prime}\left(r e^{-i \theta}\right) r d r \frac{d \theta}{2 \pi}
$$

Using this equality a simple duality argument gives

$$
J_{\infty, 2} \subset B M O \subset \text { Bloch } .
$$

$$
H^{p} \subset J_{p, p} \quad(p \leq 2)
$$

Corollary 6.3 (see [MP, Theorem 1]). $\left(H^{1}, B M O\right)=$ Bloch.

Proof. Note that we have $\left(J_{p, q}, J_{r, s}\right)=(H(p, q, 1), H(r, s, 1))$.

Using (6.4),(6.4') and (6.2) we have

$$
\text { Bloch }=(H(1,2,1), H(\infty, 2,1))=\left(J_{1,2}, J_{\infty, 2}\right) \subset\left(H^{1}, B M O\right) .
$$

Using (6.4),(6.4') and (6.1) we have

$$
\left(H^{1}, B M O\right) \subset\left(J_{1,1}, J_{\infty, \infty}\right)=(H(1,1,1), H(\infty, \infty, 1))=\text { Bloch } .
$$

Let us now give a different approach to the following result due to MacGregor and Zhu. 
Corollary 6.4 ([MZ, Theorem A]). Let $1 \leq p \leq 2$.

Then $B(n+1,1 / p) \in\left(B^{p}, H^{p}\right)$.

Proof. Observe that to show that for all $\left(a_{n}\right)$ we have

$$
\left\|\sum_{n=0}^{\infty} B(n+1,1 / p) a_{n} z^{n}\right\|_{H^{p}} \leq C\left\|\sum_{n=0}^{\infty} a_{n} z^{n}\right\|_{B^{p}},
$$

is equivalent to show that for all $\left(a_{n}\right)$ we have

$$
\left\|\sum_{n=0}^{\infty} a_{n} z^{n}\right\|_{H^{p}} \leq C\left\|\sum_{n=0}^{\infty} \frac{\Gamma(n+1+1 / p)}{\Gamma(1 / p) n !} a_{n} z^{n}\right\|_{B^{p}} .
$$

Then the proof is finished by using (6.5') together with Theorem A which implies that $f^{(1 / p)} \in H(p, p, 1 / p)$ if and only if $f \in J_{p, p}$.

We shall finish the section with some new results on multipliers on Bergman spaces. Let us first show two elementary lemmas.

Lemma 6.1. Let $1 \leq p \leq 2 \leq q<\infty$. Let $f(z)=\sum_{n=0}^{\infty} a_{n} z^{n}$.

(i) If $f \in B_{p}$ then $\left(n^{1 / 2-2 / p} a_{n}\right) \in l(2, p)$.

(ii) If $\left(n^{1 / 2-2 / q} a_{n}\right) \in l(2, q)$ then $f \in B_{q}$.

Proof. From (1.9) one has

$$
\int_{0}^{1}(1-r)^{p / 2} M_{2}^{p}\left(f^{\prime}, r\right) d r \leq C\|f\|_{H^{p}}^{p} .
$$

This implies

$$
\int_{0}^{1} \int_{0}^{1}(1-r)^{p / 2} M_{2}^{p}\left(f^{\prime}, r s\right) d r d s \leq C\|f\|_{B^{p}}^{p}
$$

Now applying Plancherel together with Lemma 2.1 one easily gets

$$
\left\|\left(n^{1 / 2-2 / p} a_{n}\right)\right\|_{2, p} \leq C\|f\|_{B^{p}} .
$$

This gives the proof of (i).

We can easily get (ii) from (i) using a dual argument from the identity

$$
\sum_{n=0}^{\infty} \frac{1}{n+1} a_{n} b_{n}=\frac{1}{2} \int_{0}^{1} \int_{0}^{2 \pi} f\left(r e^{i \theta}\right) g\left(r e^{-i \theta}\right) r d r \frac{d \theta}{2 \pi} .
$$

Remark 6.2. Lemma 6.1 might have been shown by using Corollary 3.2 which gives better estimates.

Lemma 6.2. Let $2 \leq q<\infty$ and $0<\alpha, \beta<\infty$.

$$
\text { If } \lambda_{n}=O\left(n^{\alpha-\beta-1 / q^{\prime}}\right) \text { then } \lambda^{(\beta)} \in H_{\alpha}^{q} \text {. }
$$

Proof. It is elementary to see that $\lambda_{n}=O\left(n^{\alpha-\beta-1 / q^{\prime}}\right)$ implies

$$
M_{2}\left(\lambda^{(\beta)}, r\right)=O\left(1 /(1-r)^{\alpha+1 / q-1 / 2}\right) .
$$

Now use (1.1) to get $\lambda^{(\beta)} \in H_{\alpha}^{q}$. 
Corollary 6.5 (see [V, Theorem 2]). Let $0<p \leq 2 \leq q<\infty$.

If $\lambda_{n}=O\left(n^{2 / q-2 / p}\right)$ then $\lambda_{n} \in\left(B^{p}, B^{q}\right)$

Proof. The case $1<p \leq 2$ follows trivially combining (i) and (ii).

For the case $0<p \leq 1$ we can use Theorem 5.3 to get

$$
\left(B^{p}, B^{q}\right)=\left\{\left(\lambda_{n}\right): \lambda^{(2 / p-1)} \in H_{1 / q}^{q}\right\},
$$

and then apply Lemma 6.2 with $\alpha=1 / q$ and $\beta=2 / p-1$ to show that $\lambda_{n}=$ $O\left(n^{2 / q-2 / p}\right)$ gives $\lambda^{(2 / p-1)} \in H_{1 / q}^{q}$.

In [V] Vukotic showed that for sequences $\lambda_{n}=O\left(n^{\gamma}\right)$ the exponent $\gamma=2 / q-2 / p$ was sharp in the case of multipliers in $\left(B^{p}, B^{q}\right)$. A better result in the setting of $l(p, q)$ spaces follows from Theorem 5.5.

Corollary 6.6. Let $1<p \leq 2 \leq q<\infty$. Put $\frac{1}{r}=\frac{1}{p}-\frac{1}{q}$. If $\sum_{k \in I_{n}}\left|\lambda_{k}\right|^{r}=O\left(2^{n}\right)$ then $\lambda_{n} \in\left(B^{p}, B^{q}\right)$.

\section{REFERENCES}

[ACP] J.M. Anderson, J. Clunie. Ch Pommerenke, On bloch functions and normal functions, J. Reine Angew. Math. 270 (1974), 12-37.

[AS] J.M. Anderson, A.L. Shields, Coefficient multipliers on Bloch functions, Trans. Amer. Math. Soc. 224 (1976), 256-265.

[A] S. Axler, Bergman spaces and their operators, Surveys on some recent results on Operator Theory (J.B. Conway, B.B. Morrel, editors) 171 (1988), Pitman Research Notes in Math., $1-50$.

[BST] G. Bennett, D.A. Stegenga, R.M. Timoney, Coefficients of Bloch and Lipschitz functions, Illinois J. of Math. 25 (1981), 520-531.

[B1] O. Blasco, Operators on weighted Bergman spaces and applications, Duke Math. J. 66 (1992), 443-467.

[B2] O. Blasco, Multipliers on weighted Besov spaces of analytic functions, Contemporary Math. 144 (1993), 23-33.

[D] P. Duren, Theory of $H_{p}$-spaces (1970), Academic Press, New York.

[DS1] P. Duren, A.L. Shields, Coefficient multipliers of $H^{p}$ and $B^{p}$ spaces, Pacific J. Math. 32 (1970), 69-78.

[DS2] P. Duren, A.L. Shields, Properties of $H^{p}(0<p<1)$ and its containing Banach space, Trans. Amer. Math. Soc. 141 (1969), 255-262.

[DRS] P.L. Duren, B.W. Romberg, A.L. Shields, Linear functionals on $H_{p}$-spaces with $0<p<1$, J. Reigne Angew. Math. 238 (1969), 32-60.

[F1] T.M. Flett, The dual of an inequality of Hardy and Littlewood and some related inequalities, J. Math. Anal. and Appl. 38 (1972), 746-765.

[F2] T.M. Flett, Lipschitz spaces of functions on the circle and the disc, J. Math. Anal. and Appl. 39 (1972), 125-158.

[F3] T.M. Flett, On the rate of growth of mean valeus of holomorphic and harmonic functions, Proc. London Math. Soc. 20 (1970), 749-768.

[HL1] G.H. Hardy, J.E. Littlewood, Some properties of fractional integrals II, Math. Z. 34 (1932), 403-439.

[HL2] G.H. Hardy, J.E. Littlewood, Theorems concerning mean values of analytic or harmonic functions, Quart. J. Math. 12 (1941), 221-256.

[JP] M. Jevtic, M. Pavlovic, On multipliers from $H^{p}$ to $l^{q}, 0<q<p<1$, Arch. Math. 56 (1991), 174-180.

[Ka] Y. Katnetzon, An introduction to Harmonic Analysis, John Wiley and sons, New York, 1968 . 
[K] C. N. Kellogg, An extension of the Hausdorff-Young Theorem, Michigan. Math. J. 18 (1971), 121-127.

[Ki] S.V. Kisliakov, Fourier coefficients of boundary values of analytic functions on the disc and the bidisc, Trudy Mat. Inst. Steklov 155 (1981), 77-91.

[LP] J.E. Littlewood, R.E.A.C. Paley, Theorems on Fourier series and power series, J. London Math. Soc 42 (1931), 52-89.

[MZ] T. MacGregor, K. Zhu, Coefficient multipliers between Bergman and Hardy spaces, Preprint.

[Ma] M. Marzuq, Linear functionals on some weighted Bergman spaces, Bull. Austral. Math. Soc. 42 (1990), 417-425.

[MP1] M. Mateljevic, M. Pavlovic, $L^{p}$ behaviour of power series with positive coefficients and Hardy spaces, Proc. Amer. Math. Soc. 87 (1983), 309-316.

[MP2] M. Mateljevic, M. Pavlovic, $L^{p}$ behaviour of the integral means of analytic functions, Studia Math. 77 (1984), 219-237.

[MP3] M. Mateljevic, M. Pavlovic, Multipliers of $H^{p}$ and $B M O$, Pacific J. Math. 146 (1990), 71-84.

[M] A. Matheson, A Multipliers theorem for analytic functions of slow mean growth, Proc. Amer. Math. Soc. 77 (1979), 53-57.

[S1] W. Sledd, Some results about spaces of analytic functions introduced by Hardy and Littlewood, J. London Math. Soc. 2 (1974), 328-336.

[S2] W. Sledd, On multipliers of $H^{p}$ spaces, Indiana Univ. Math. J. 27 (1978), 797-803.

[Sh] J.H. Shapiro, Mackey topologies, reproducing kernels and diagonal maps on the Hardy and Bergman spaces, Duke Math. J. 43 (1976), 187-202.

[SW] A.L.Shields, D.L. Williams, Bounded projections, duality and multipliers in spaces of analytic functions, Trans. Amer. Math. Soc. 162, (1971), 287-302.

[St] D. Stegenga, Multipliers of the Dirichlet space, Illinois J. Math. 24 (1980), 113-139.

[V] D. Vukotic, On the coefficient multipliers of Bergman spaces, Preprint.

[W] P. Wojtaszcyk, Multipliers into Bergman spaces and Nevalinna class, Canad. Math. Bull. 33 (1990), 151-161.

[Z] K. Zhu, Operator theory in function spaces, Marcel Dekker, Inc., New York, 1990.

[Zy] A. Zygmund, Trigonometric series, Cambrigde Univ. Press., New York, 1959.

Oscar Blasco. Departamento de Análisis Matemático, Universidad de Valencia, 46100 Burjassot (Valencia), Spain.

E-mail address: Blascod@mac.uv.es 